\title{
Splines and pseudo-inverses
}

RAIRO - Analyse numérique, tome 12, no 4 (1978), p. 313-324.

$<$ http://www.numdam.org/item?id=M2AN_1978_12_4_313_0>

(C) AFCET, 1978, tous droits réservés.

L'accès aux archives de la revue «RAIRO - Analyse numérique » implique l'accord avec les conditions générales d'utilisation (http://www.numdam.org/ legal.php). Toute utilisation commerciale ou impression systématique est constitutive d'une infraction pénale. Toute copie ou impression de ce fichier doit contenir la présente mention de copyright.

\section{Numdam}

Article numérisé dans le cadre du programme

Numérisation de documents anciens mathématiques

http://www.numdam.org/ 


\title{
SPLINES AND PSEUDO-INVERSES ( $\left.{ }^{*}\right)$
}

\author{
by F. J. Delvos $\left({ }^{1}\right)$ \\ Communiqué par P J LAURENT
}

\begin{abstract}
We introduce abstract interpolating splines of minimum norm by using the concept of restricted pseudo-inverse of Minamide and Nakamura We propose a certain invariance relation which generalizes the Golomb-Weinberger-Sard method As an application we consider abstract tensor product splines.
\end{abstract}

\section{INTRODUCTION}

The pseudo-inverse finds a wide range of applications to statistics, optımal control theory, curve fitting etc. Application of the theory to various fields rests upon the "best approximate solution property" of the pseudo-inverse which is closely associated with orthogonal projectors in a Hilbert space. In this paper we will apply the concept of pseudo-inverse to abstract splines as started by Laurent [9].

In section 1 the properties of the pseudo-inverse are summarized and certain lemmas are proved. In section 2 interpolating splines of minimum norm are defined using the concept of restricted pseudo-inverse introduced by MinamideNakamura [10]. The structure of splines of minimum norm is discussed if a certain invariance relation is valid. In the last section, the theory is applied to abstract tensor product splines.

\section{NOTE ON PSEUDO-INVERSES}

We adopt the notations of Minamide-Nakamura [10]. Let $H_{1}$ and $H_{2}$ be Hilbert spaces and $L\left(H_{1}, H_{2}\right)$ the set of continuous linear operators $T$ from $H_{1}$ into $H_{2}$ with closed range in which case $T$ is called normally solvable. For each

(*) Reçu janvier 1978.

$\left({ }^{1}\right)$ Fachbereıch Mathematık, Unıversity of Siegen, D 5900 Siegen 21, Allemagne (Républıque Fédérale).

R A I R O Analyse numérıque/Numenical Analysıs, vol 12, nº 4, 1978 
continuous linear operator $T: H_{1} \rightarrow H_{2}$ let $R(T)$ and $N(T)$ denote the range and the kernel of $T$, respectively. The following lemma 1.1 can be found in [11]:

Lemma $1.1:$ Let $\gamma(T)$ be the (extended) real number defined by

$\gamma(T)=\inf \left\{\|T x\| /\|x\|: x \in N(T)^{\perp}, x \neq 0\right\}$,

$\gamma(0):=\infty$.

Then $T$ is normally solvable iff $\gamma(T)>0$.

[ $N(T)^{\perp}$ denotes the orthogonal complement of $N(T)$.] The orthogonal projector on a subspace $L$ is denoted by $P_{L}$. In particular, the projectors $P_{R(T)}$, $P_{N(T)^{\perp}}$ can be represented in terms of the pseudo-inverse $T^{+}$(Moore's definition [11]):

$$
\begin{aligned}
& P_{R(T)}=T T^{+}, \\
& P_{N(T)^{\perp}}=T^{+} T .
\end{aligned}
$$

For a normally solvable operator $T$ the pseudo-inverse $T^{+} \in L\left(H_{2}, H_{1}\right)$ may also be defined as the unique solution $X$ of the Penrose equations [11):

$X T X=X$,

$T X T=T$,

$(T X)^{*}=T X$,

$(X T)^{*}=X T$.

The pseudo-inverse $T^{+}$enjoys the "best approximate solution property" for the equation $T u=\xi$ :

$\left\|T T^{+} \xi-\xi\right\| \leqq\|T u-\xi\| \quad\left(u \in H_{1}\right)$,
$\|T u-\xi\|=\left\|T T^{+} \xi-\xi\right\| \Rightarrow\left\|T^{+} \xi\right\| \leqq\|u\|$.

In later discussion we shall use the relations

$T^{+}=\left(T^{*} T\right)^{+} T^{*}$,

$N\left(T^{+}\right)=N\left(T^{*}\right)=R(T)^{\perp}$,

$R\left(T^{+}\right)=R\left(T^{*}\right)=N(T)^{\perp}$,

$R\left(T^{*} T\right)=N\left(T^{*} T\right)^{\perp}=N(T)^{\perp}$. (see [4]).

Let $H_{3}$ be a Hilbert space and $S \in L\left(H_{1}, H_{3}\right)$ be a normally solvable operator. We consider the product operator $T \times S$ from $H_{1}$ into $H_{2} \times H_{3}$ which is 
defined by

$T \times S(x)=(T x, S x)$.

Here $\mathrm{H}_{2} \times \mathrm{H}_{3}$ is a product Hilbert space equipped with the usual inner product.

Lemma 1.2 [10]: The product operator $T \times S$ is normally solvable iff $T P_{N(S)}$ (respectively $S P_{N(T)}$ ) is normally solvable.

This is the case if, for instance, the inclusion $N(T) \subset N(S)$ [respectively $N(S) \subset N(T)$ ] is valid. This can be extended to

Lemma 1.3: Suppose that the projectors $P_{N(T)}, P_{N(S)}$ commute:

$P_{N(T)} P_{N(S)}=P_{N(S)} P_{N(T)}$.

Then the operator $T P_{N(S)}$ is normally solvable.

Proof: We prove lemma 1.3 by applying lemma 1.1 to the operator $T P_{N(S)}$. Taking into account (1.3) and (1.14) we have

$$
\begin{aligned}
N\left(T P_{N(S)}\right) & =N\left(T^{+} T P_{N(S)}\right) \\
& =N\left(P_{N(T)^{\perp}} P_{N(S)}\right) \\
& =N\left(P_{N(T)^{\perp} \cap N(S)}\right)
\end{aligned}
$$

whence

$N\left(T P_{N(S)}\right)^{\perp}=N(T)^{\perp} \cap N(S)$.

Now we can conclude for each $x \in N\left(T P_{N(S)}\right)^{\perp}, x \neq 0$ :

$\left\|T P_{N(S)} x\right\| /\|x\|=\|T x\| /\|x\| \geqq \gamma(T)$,

i. e. we have

$\gamma\left(T P_{N(S)}\right) \gtrless \gamma(T)>0$.

This completes the proof of lemma 1.3.

Note that lemma 1.3 is applicable if the inclusion

$N(T) \subset N(S)^{\perp}$

holds.

LEMMA 1.4: Suppose that the invariance relation

$T^{*} T P_{N(\mathcal{S})^{\perp}}=P_{N(S) \perp} T^{*} T$

is valid. Then the projectors $P_{N(T)}, P_{N(S)}$ commute, i. e. the operator $T P_{N(S)}$ is normally solvable. 
Proof: Taking into account (1.3), (1.12), (1.5), (1.17) we obtain

$$
\begin{aligned}
& T^{*} T P_{N\left(S \perp^{\perp}\right.}=P_{N(S)^{\perp}} T^{*} T, \\
& \left(T^{*} T\right)^{+}\left(T^{*} T\right) P_{N(S)^{\perp}}=\left(T^{*} T\right)^{+} P_{N(S)^{\perp}}\left(T^{*} T\right), \\
& P_{N(T)^{\perp}} P_{N(S)^{\perp}}\left(T^{*} T\right)^{+}=\left(T^{*} T\right)^{+} P_{N(S)^{\perp}}\left(T^{*} T\right)\left(T^{*} T\right)^{+}, \\
& P_{N(T)^{\perp}} P_{N(S)^{\perp}}\left(T^{*} T\right)^{+}\left(T^{*} T\right)=\left(T^{*} T\right)^{+} P_{N(S)^{\perp}}\left(T^{*} T\right)\left(T^{*} T\right)^{+}\left(T^{*} T\right), \\
& P_{N(T)^{\perp}} P_{N(S)^{\perp}} P_{N(T)^{\perp}}=\left(T^{*} T\right)^{+} P_{N(S)^{\perp}}\left(T^{*} T\right), \\
& P_{N(T)^{\perp}} P_{N(S)^{\perp}} P_{N(T)^{\perp}}=\left(T^{*} T\right)^{+}\left(T^{*} T\right) P_{N(S)^{\perp},}
\end{aligned}
$$

i. e. we have

$$
P:=P_{N(T)^{\perp}} P_{N(S)^{\perp}}=P_{N(T)^{\perp}} P_{N(S)^{\perp}} P_{N(T)^{\perp}}=P^{*} .
$$

Since

$$
\begin{aligned}
P^{2} & =P_{N(T)^{\perp}} P_{N(S)^{\perp}} P_{N(T)^{\perp}} P_{N(S)^{\perp}} \\
& =P_{N(T)^{\perp}} P_{N(S)^{\perp}} P_{N(S)^{\perp}} \\
& =P
\end{aligned}
$$

the projectors $P_{N(T)^{\perp}}, P_{N(S)^{\perp}}$ commute. This completes the proof of lemma 1.4. Laurent [9] used the pseudo-inverse $(T \times S)^{+}$to construct smoothing splines by applying the best approximate solution property to the equation

$T \times S(u)=(0, \eta)$.

ivinamide and Nakamura [10] introduced the concept of restricted pseudoinverse which posseses a "constrained best approximate solution property" and which has applications to certain constrained minimization problems. In view of a result of Hall [6] the restricted pseudo-inverse $T_{S}^{+}$of $T$ with respect to $N(S)$ may be defined as

$T_{S}^{+}:=\left(T P_{N(S)}\right)^{+}$.

Here $T P_{N(s)}$ is supposed to be normally solvable. The restricted pseudoinverse $T_{S}^{+}$enjoys the "best approximate solution property" for the ordered system of equations

$T u=\xi, \quad S u=\eta$.

Theorem 1.1 [10]: For $\eta \in R(S)$ and $\xi \in H_{2}$ let

$u_{0}=T_{S}^{+}\left(\xi-T S^{+} \eta\right)+S^{+} \eta$. 
Then $u_{0}$ is the unique element in $H_{1}$ satisfying

$S u_{0}=\eta$

so that

$\left\|T u_{0}-\xi\right\| \leqq\|T u-\xi\| \quad(S u=\eta)$,

$\left\|u_{0}\right\| \leqq\|u\| \quad\left(S u=\eta,\|T u-\xi\|=\left\|T u_{0}-\xi\right\|\right)$.

We shall apply the "constrained best approximate solution property" to the construction of interpolating splines.

The following lemma 1.5 will be used in later discussion.

Lemma 1.5: Suppose that the projectors $P_{N(T)}, P_{N(S)}$ commute. Then the restricted pseudo-inverse $T_{s}^{+}$is given by

$T_{S}^{+}=T^{+} P_{R\left(T P_{N(S)}\right.}$.

Proof: It follows from lemma 1.3 and (1.2) that

$P_{R\left(T P_{N(S)}\right)}=T P_{N(S)}\left(T P_{N(S)}\right)^{+}$.

Taking into account (1.25), (1.3), (1.14), (1.15), (1.11) and (1.19) we obtain

$$
\begin{aligned}
T^{+} P_{R\left(T P_{N(S)}\right)} & =T^{+} T P_{N(S)}\left(T P_{N(S)}\right)^{+} \\
& =P_{N(T)^{\perp}} P_{N(S)}\left(T P_{N(S)}\right)^{+} \\
& =P_{N(T)^{\perp} \cap N(S)}\left(T P_{N(S)}\right)^{+} \\
& =P_{N\left(T P_{N(S)}\right)^{\perp}}\left(T P_{N(S)}\right)^{+} \\
& =P_{R\left(\left(T P_{\left.N(S)^{+}\right)}\right.\right.}\left(T P_{N(S)}\right)^{+} \\
& =\left(T P_{N(S)}\right)^{+} \\
& =T_{S}^{+} .
\end{aligned}
$$

This completes the proof of lemma 1.5.

\section{SPLINES OF MINIMUM NORM}

Throughout this section we shall assume that the operators $T \in L\left(H_{1}, H_{2}\right), \quad S \in L\left(H_{1}, H_{3}\right)$,

$T \times S \in L\left(H_{1}, H_{2} \times H_{3}\right)$

are normally solvable. The space of splines relative to $T, S$ is defined as

$\operatorname{Sp}(T, S):=\left\{x \in H_{1}:(T x, T y)=0(y \in N(S))\right\}$.

vol. $12, n^{\circ} 4,1978$ 
Our first objective is to derive a simple characterization of $\operatorname{Sp}(T, S)$ using the concept of restricted pseudo-inverse.

THeOREm 2.1: The space of splines relative to $T, S$ is the kernel of the operator $T_{S}^{+} T$ :

$\operatorname{Sp}(T, S)=N\left(T_{S}^{+} T\right)$.

Proof: We have

$$
\begin{aligned}
x \in \operatorname{Sp}(T, S) & \Leftrightarrow(T x, T y)=0 \quad(y \in N(S)) \\
& \Leftrightarrow\left(T x, T P_{N(S)} y\right)=0 \quad\left(y \in H_{1}\right) \\
& \Leftrightarrow T x \in R\left(T P_{N(S)}\right)^{\perp} .
\end{aligned}
$$

Taking into account (1.19), (1.10) we obtain

$x \in \operatorname{Sp}(T, S) \Leftrightarrow T x \in N\left(T_{S}^{+}\right) \Leftrightarrow x \in N\left(T_{S}^{+} T\right)$,

i. e. we have (2.3).

Our next purpose is to construct interpolating splines. Putting $\xi=0$ in theorem 1.1 we obtain

THEOREM 2.2: For $\eta \in R(S)$ let

$u_{0}=S^{+} \eta-T_{S}^{+} T S^{+} \eta$.

Then $u_{0}$ is the best approximate solution of

$T u=0, \quad S u=\eta$,

i. e. $u_{0}$ is the unique element in $H_{1}$ so that

$S u_{0}=\eta$,

$\left\|T u_{0}\right\| \leqq\|T u\| \quad(S u=\eta)$

$\left\|u_{0}\right\| \leqq\|u\| \quad\left(S u=\eta,\|T u\|=\left\|T u_{0}\right\|\right)$.

Because of (2.6), (2.7) the element $u_{0}$ is an interpolating spline relative to $T, S$. In view of $(2.8) u_{0}$ is called the interpolating spline of minimum norm relative to $T, S$, $\eta \in R(S)$. If the uniqueness relation is valid

$N(T) \cap N(S)=\{0\}$,

the relation (2.8) is redundant.

It is almost obvious that the operator $P_{S}$ of minimum norm spline interpolation is given by

$P_{S}=S^{+} S-T_{S}^{+} T S^{+} S$. 
Furthermore, the set

$\mathrm{Sp}_{0}(T, S)=R\left(P_{S}\right)$

will be called the space of interpolating splines of minimum norm. Some properties of $P_{S}$ are listed in theorem 2.3.

THEOREM 2.3: The following relations are valid:

$T_{S}^{+} T P_{S}=0, \quad\left[\right.$ i. e. $\left.\mathrm{Sp}_{0}(T, S) \subset \mathrm{Sp}(T, S)\right]$,

$N\left(P_{S}\right)=N(S)$,

$P_{S} P_{S}=P_{S}$.

Proof: It follows from (1.19), (1.11) that

$$
\begin{aligned}
R\left(T_{S}^{+}\right) & =R\left(\left(T P_{N(S)}\right)^{*}\right) \\
& =R\left(P_{N(S)} T^{*}\right) \\
& \subset N(S)
\end{aligned}
$$

whence

$P_{N(S)} T_{S}^{+}=T_{S}^{+}=\left(T P_{N(S)}\right)^{+}=P_{N(S)}\left(T P_{N(S)}\right)^{+}$.

Taking into account (2.10), (2.15), (1.4) we obtain

$$
\begin{aligned}
T_{S}^{+} T P_{S}= & \left(T P_{N(S)}\right)^{+} T P_{N(S)^{\perp}} \\
& -\left(T P_{N(S)}\right)^{+} T\left(T P_{N(S)}\right)^{+} T P_{N(S)^{\perp}} \\
= & \left(T P_{N(S)}\right)^{+} T P_{N(S)^{\perp}} \\
& -\left(T P_{N(S)}\right)^{+}\left(T P_{N(S)}\right)\left(T P_{N(S)}\right)^{+} T P_{N(S)^{\perp}} \\
= & \left(T P_{N(S)}\right)^{+} T P_{N(S)^{\perp}}-\left(T P_{N(S)}\right)^{+} T P_{N(S)^{\perp}} \\
= & 0 .
\end{aligned}
$$

Thus, the relation (2.12) is proved.

It follows from the definition (2.10) that

$N(S) \subset N\left(P_{S}\right)$.

On the other hand, it follows from (2.15) that

$S P_{S}=S S^{+} S-S T_{S}^{+} T S^{+} S=S S^{+} S$

whence

$S P_{S}=S$.

vol. $12, \mathrm{n}^{\circ} 4,1978$ 
This proves together with (2.16) the relation (2.13).

Finally, the equality (2.14) follows again from (2.15):

$$
\begin{aligned}
P_{S} P_{S}= & S^{+} S S^{+} S-S^{+} S T_{S}^{+} T S^{+} S \\
& -T_{S}^{+} T S^{+} S S^{+} S+T_{S}^{+} T S^{+} S T_{S}^{+} T S^{+} S \\
= & S^{+} S-T_{S}^{+} T S^{+} S \\
= & P_{S} .
\end{aligned}
$$

This completes the proof of theorem 2.3.

In view of theorem 2.3 the operator $P_{S}$ will be called the spline projector relative to $T, S$.

Our next objective is to study the splines of minimum norm $\operatorname{Sp}_{0}(T, S)$ if the invariance relation

$T^{*} T P_{N(S) \perp}=P_{N(S)} T^{*} T$

is valid (see lemma 1.4). Our main result is contained in

THEOREM 2.4: Suppose that the invariance relation (2.18) holds. Then the interpolating spline of minimum norm relative to $T, S, \eta \in R(S)$ is given by $u_{0}=S^{+} \eta$.

Furthermore, the following relations are true:

$P_{S}=S^{+} S=P_{N(S)^{\perp}}$,

$\mathrm{Sp}_{0}(T, S)=N(S)^{\perp}$.

Proof: Since

$P_{S}=S^{+} S-T_{S}^{+} T S^{+} S$

it suffices to show that

$T_{S}^{+} T P_{N(S)}^{\perp}=0$.

Note that the invariance relation (2.18) implies that the operator $T P_{N(S)}$ is normally solvable (see lemma 1.4 and 1.3). Taking into account (1.24), (1.2), (1.9), (2.18) we obtain

$$
\begin{aligned}
T_{S}^{+} T P_{N(S)^{\perp}} & =T^{+} P_{R\left(T P_{N(S)}\right.} T P_{N(S)^{\perp}} \\
& =T^{+}\left(T P_{N(S)}\right)\left(T P_{N(S)}\right)^{+} T P_{N(S)^{\perp}} \\
& =T^{+} T P_{N(S)}\left(\left(T P_{N(S)}\right)^{*}\left(T P_{N(S)}\right)\right)^{+}\left(T P_{N(S)}\right)^{*} T P_{N(S)^{\perp}} \\
& =T^{+} T P_{N(S)}\left(P_{N(S)} T^{*} T P_{N(S)}\right)^{+} P_{N(S)} T^{*} T P_{N(S)^{\perp}} \\
& =0 .
\end{aligned}
$$


This proves (2.20) and (2.21).

The relation (2.19) is a consequence of (2.4), (1.4), (1.3), (2.22):

$u_{0}=S^{+} \eta-T_{S}^{+} T S^{+} \eta=S^{+} \eta-T_{S}^{+} T P_{N(S)}^{\perp} S^{+} \eta=S^{+} \eta$.

Corollary 2.1 [9]: Suppose that $H_{1}$ carries the Golomb-Weinberger-Sard scalar product [12]:

$(x, y)_{1}=(T x, T y)_{2}+(S x, S y)_{3} \quad\left(x, y \in H_{1}\right)$.

Then the interpolating spline relative to $T, S, \eta \in R(S)$ is given by

$u_{0}=S^{+} \eta$.

Proof: It is sufficient to show that the invariance relation (2.18) is valid. For arbitrary $x, y \in H_{1}$, we have

$$
\begin{aligned}
\left(P_{N(S)^{\perp}} T^{*} T(x), y\right)_{1} & =\left(T^{*} T(x), P_{N(S)^{\perp}}(x)\right)_{1} \\
& =\left(T(x), T P_{N(S)^{\perp}}(y)\right)_{2} \\
& =\left(x, P_{N(S)^{\perp}}(y)\right)_{1}-\left(S x, S P_{N(S)^{\perp}}(y)\right)_{3} \\
& =\left(P_{N(S)^{\perp}}(x), y\right)_{1}-(S x, S y)_{3} \\
& =\left(T P_{N(S)^{\perp}}(x), T y\right)_{2} \\
& =\left(T^{*} T P_{N(S)^{\perp}}(x), y\right)_{1},
\end{aligned}
$$

i. e. the invariance relation (2.18) holds.

REMARK: It should be noted that the assumption of corollary 2.1 implies the uniqueness relation

$N(T) \cap N(S)=\{0\}$.

The following corollary does not depend on the uniqueness condition.

Corollary 2.2: Suppose that the projectors $P_{N(T)}, P_{N(S)}$ commute and that $T$ is a partial isometry:

$P_{N(T)} P_{N(S)}=P_{N(S)} P_{N(T)}$,

$T^{+}=T^{*}$.

vol. $12, \mathrm{n}^{\circ} 4,1978$ 
Then the interpolating spline of minimum norm relative to $T, S, \eta \in R(S)$ is given by $u_{0}=S^{+} \eta$.

Proof: Again, the invariance relation (2.18) follows immediately from (2.26), (2.27):

$$
\begin{aligned}
T^{*} T P_{N(S)^{\perp}} & =T^{+} T P_{N(S)^{\perp}} \\
& =P_{N(T)^{\perp}} P_{N(S)^{\perp}} \\
& =P_{N(S)^{\perp}} P_{N(T)^{\perp}} \\
& =P_{N(S)^{\perp}} T^{*} T .
\end{aligned}
$$

\section{APPLICATION TO TENSOR PRODUCT SPLINES}

In this section, we shall exhibit an application of the theory to abstract tensor product splines. For tensor product splines, the uniqueness relation is not valid in general. We shall consider tensor product operators

$T=T^{\prime} \otimes T^{\prime \prime}, \quad S=S^{\prime} \otimes S^{\prime \prime}$

where

$T^{\prime} \in L\left(H_{1}^{\prime}, H_{2}^{\prime}\right), \quad T^{\prime \prime} \in L\left(H_{1}^{\prime \prime}, H_{2}^{\prime \prime}\right)$,

$S^{\prime} \in L\left(H_{1}^{\prime}, H_{3}^{\prime}\right), \quad S^{\prime \prime} \in L\left(H_{1}^{\prime \prime}, H_{3}^{\prime \prime}\right)$,

are normally solvable operators. (For tensor products of Hilbert spaces and linear operators we refer to [1].)

Our first theorem 3.1 extends to normally solvable operators a result which is known for invertible operators and for arbitrary matrices $([1,5])$.

Theorem 3.1: Suppose that $T^{\prime}, T^{\prime \prime}$ are normally solvable. Then the tensor product operator $T^{\prime} \otimes T^{\prime \prime}$ is normally sovable so that

$\left(T^{\prime} \otimes T^{\prime \prime}\right)^{+}=T^{\prime+} \otimes T^{\prime \prime+}$.

Proof: Let us first show that $T^{\prime} \otimes T^{\prime \prime}$ is normally solvable. Since $T^{\prime}, T^{\prime \prime}$ are normally solvable we have

$$
\begin{aligned}
& T^{\prime+} T^{\prime}=P_{N(T)^{\perp}}, \quad T^{\prime \prime}+T^{\prime \prime}=P_{N\left(T^{\prime \prime}\right)^{\perp}}, \\
& T^{\prime}=T^{\prime} P_{N\left(T^{\prime}\right)^{\perp}}, \quad T^{\prime \prime}=T^{\prime \prime} P_{N\left(T^{\prime \prime}\right)^{\perp}}
\end{aligned}
$$


Then

$\left(T^{\prime+} \otimes T^{\prime \prime+}\right)\left(T^{\prime} \otimes T^{\prime \prime}\right)=P_{N\left(T^{\prime}\right) \perp} \otimes P_{N\left(T^{\prime \prime}\right)^{\perp}}$

$T^{\prime} \otimes T^{\prime \prime}=\left(T^{\prime} \otimes T^{\prime \prime}\right)\left(P_{N\left(T^{\prime}\right)} \otimes P_{N\left(T^{\prime \prime}, \perp\right)}\right.$

whence

$N\left(T^{\prime} \otimes T^{\prime \prime}\right)=N\left(P_{N\left(T^{\prime}\right)} \otimes P_{N\left(T^{\prime \prime}\right)}\right)$.

Since $P_{N\left(T_{0}\right)^{\perp}} \otimes P_{N\left(T^{\prime \prime}\right) \perp}^{\perp}$ is an orthogonal projector we have [3]:

$P_{N\left(T^{\prime} \otimes T^{\prime \prime}\right)^{\perp}}=P_{N\left(T^{\prime}\right)^{\perp}} \otimes P_{N\left(T^{\prime \prime}\right)^{1}}$.

To show that $T^{\prime} \otimes T^{\prime \prime}$ is normally solvable we may assume without loss of generality that $T^{\prime} \otimes T^{\prime \prime} \neq 0$, i. e. we have $T^{\prime} \neq 0^{\prime}, T^{\prime \prime} \neq 0^{\prime \prime}$ and therefore $\left\|T^{\prime+} \otimes T^{\prime \prime}\right\|=\left\|T^{\prime+}\right\| \cdot\left\|T^{\prime \prime}\right\|>0$.

Taking into account (3.9), (3.6) we can conclude for $x \in N\left(T^{\prime} \otimes T^{\prime \prime}\right)^{\perp}, x \neq 0$ :

$$
\begin{aligned}
\left\|T^{\prime} \otimes T^{\prime \prime}(x)\right\| /\|x\| & =\left\|T^{\prime} \otimes T^{\prime \prime}(x)\right\| /\left\|P_{N\left(T^{\prime \prime} \otimes T^{\prime \prime}\right)^{\perp}}(x)\right\| \\
& =\left\|T^{\prime} \otimes T^{\prime \prime}(x)\right\| / \|\left(P_{N\left(T^{\prime}\right)} \otimes P_{\left.N\left(T^{\prime \prime}\right) \perp\right)}(x) \|\right. \\
& =\left\|T^{\prime} \otimes T^{\prime \prime}(x)\right\| /\left\|\left(T^{\prime+} \otimes T^{\prime \prime}\right)\left(T^{\prime} \otimes T^{\prime \prime}\right)(x)\right\| \\
& \geqq\left\|T^{\prime+} \otimes T^{\prime \prime}\right\|^{-1}
\end{aligned}
$$

whence

$\gamma\left(T^{\prime} \otimes T^{\prime \prime}\right) \geqq\left\|T^{\prime+} \otimes T^{\prime \prime+}\right\|^{-1}>0$,

i. e. the operator $T^{\prime} \otimes T^{\prime \prime}$ is normally solvable (see lemma 1.1).

The equality (3.3):

$\left(T^{\prime} \otimes T^{\prime \prime}\right)^{+}=T^{\prime+} \otimes T^{\prime \prime}+$

is established by showing that $T^{\prime+} \otimes T^{\prime \prime+}$ satisfies the Penrose equations (1.4)(1.7) with $T=T^{\prime} \otimes T^{\prime \prime}$. The proof is purely algebraic as in the case of matrices [5].

We are now able to state the main result of this section.

THEOREM 3.2: Suppose that

$T^{\prime *} T^{\prime} P_{N\left(S^{\prime} \perp\right.}=P_{N\left(S^{\prime}\right)^{\perp}} T^{*} T^{\prime}$,

$T^{\prime \prime *} T^{\prime \prime} P_{N\left(S^{\prime \prime}\right) \perp}=P_{N\left(S^{\prime \prime}\right) \perp} T^{\prime \prime *} T^{\prime \prime}$.

Then

$u_{0}=S^{\prime+} \otimes S^{\prime \prime+}(\eta)$

is the interpolating tensor product spline of minimum norm [relative to $T^{\prime} \otimes T^{\prime \prime}$, $S^{\prime} \otimes S^{\prime \prime}$ and $\left.\eta \in R\left(S^{\prime} \otimes S^{\prime \prime}\right)\right]$. Furthermore, we have

$P_{S^{\prime} \otimes S^{\prime \prime}}=P_{S^{\prime}} \otimes P_{S^{\prime \prime}}$

$\mathrm{Sp}_{0}\left(T^{\prime} \otimes T^{\prime \prime}, S^{\prime} \otimes S^{\prime \prime}\right)=\mathrm{Sp}_{0}\left(T^{\prime}, S^{\prime}\right) \otimes \mathrm{Sp}_{0}\left(T^{\prime \prime}, S^{\prime \prime}\right)$.

vol. $12, n^{\circ} 4,1978$ 
Proof: In view of theorem 3.1, 2.4, and (3.9) it is sufficient to show that the invariance relation is valid for the tensor product operators $T^{\prime} \otimes T^{\prime \prime}, S^{\prime} \otimes S^{\prime \prime}$ : $\left(T^{\prime} \otimes T^{\prime \prime}\right)^{*}\left(T^{\prime} \otimes T^{\prime \prime}\right) P_{N\left(S^{\prime} \otimes S^{\prime \prime}\right)^{\perp}}=P_{N\left(S^{\prime} \otimes S^{\prime \prime}\right)^{\perp}}\left(T^{\prime} \otimes T^{\prime \prime}\right)^{*}\left(T^{\prime} \otimes T^{\prime \prime}\right)$.

Taking into account (3.9), (3.12) we obtain

$$
\begin{aligned}
\left(T^{\prime} \otimes T^{\prime \prime}\right)^{*}\left(T^{\prime} \otimes T^{\prime \prime}\right) P_{N\left(S^{\prime} \otimes S^{\prime \prime}\right)^{\perp}} & \\
& =\left(T^{*} T^{\prime} \otimes T^{\prime \prime} * T^{\prime \prime}\right)\left(P_{N\left(S^{\prime}\right)^{\perp}} \otimes P_{N\left(S^{\prime}\right)}\right) \\
& =\left(T^{\prime *} T^{\prime} P_{N\left(S^{\prime}\right) \perp}\right) \otimes\left(T^{\prime \prime *} T^{\prime \prime} P_{N\left(S^{\prime \prime}\right)^{\perp}}\right) \\
& =\left(P_{N\left(S^{\prime}\right)^{\perp}} T^{\prime *} T^{\prime}\right) \otimes\left(P_{N\left(S^{\prime \prime}\right) \perp} T^{\prime \prime *} T^{\prime \prime}\right) \\
& =P_{N\left(S^{\prime} \otimes S^{\prime}\right)^{\perp}}\left(T^{\prime} \otimes T^{\prime \prime}\right)^{*}\left(T^{\prime} \otimes T^{\prime \prime}\right) .
\end{aligned}
$$

This completes the proof of theorem 3.2.

REMARK: Since

$N\left(T^{\prime}\right) \otimes N\left(S^{\prime \prime}\right)+N\left(S^{\prime}\right) \otimes N\left(T^{\prime \prime}\right) \subset N\left(T^{\prime} \otimes T^{\prime \prime}\right) \cap N\left(S^{\prime} \otimes S^{\prime \prime}\right)$

the uniqueness relation is not valid for tensor product splines in general. Thus, the relation $P_{S^{\prime} \otimes S^{\prime \prime}}=P_{S^{\prime}} \otimes P_{S^{\prime \prime}}$ is often used to define tensor product splines $[2,3,7,8]$.

\section{REFERENCES}

1. J. M. Berezanskir, Expansions in Eigenfunctions of Selfadjoint Operators, Amer. Math. Soc., Providence, R. I., 1968.

2. F. J. Delvos and H. Posdorf, On Optimal Tensor Product Approximation, J. Approximation Theory, Vol. 18, 1976, pp. 99-107.

3. F. J. Delvos and K. H. Schlosser, Das Tensorprodukt-Schema von Spline Systemen in Spline-Funktionen K. Böhmer, G. Meinardus, W. Schempp, Mannheim, WienZürich, Bibliographisches Institut, 1974.

4. C. A. Desoen and B. H. Whàlen, A note on Psendo inverses, J. Soc. Industr. Math., Vol. 11, 1963, pp. 442-447.

5. T. N. E. Greville, Note on Fitting Functions of Several Variables, J. Soc. Industr. Appl. Math., Vol. 9, 1961, pp. 109-115.

6. F. J. HALL, Generalized Inverses of a Bordered Matrix of Operators, S.I.A.M. J. Appl. Math., Vol. 29, 1975, pp. 152-163.

7. W. Haussmann, On Multivariate Spline Systems, J. Approximation Theory, Vol. 11, 1974, pp. 285-305.

8. W. HaUsSMANN and H. J. MÜNCH, On the Construction of Multivariate Spline Systems in Approximation Theory, G. G. Lorentz, New York, London, Academic Press, 1973, pp. 337-378.

9. P. L. Laurent, Approximation et optimisation, Paris, Hermann, 1972.

10. N. Minamide and K. Nakamura, A Restricted Pseudo-Inverse and its Application to Constrained minima, S.I.A.M. J. Appl. Math., Vol. 19, 1970, pp 167-177.

11. W. Petryshyn, On Generalized Inverses and on the Uniform Convergence of $(I-\beta K)^{n}$ with Application to Iterative Methods, J. Math. Anal. and Appl., Vol. 18, 1967, pp. 417-439.

12. A. SARD, Optimal Approximation, J. Funct. Anal., Vol. 1, 1967, pp. 222-244. 